\title{
Antagonistic activity of Lactobacillus reuteri strains isolated from different niches against food bacteria and its biopreservative application along with nisin in milk system
}

\author{
Diwas Pradhan ${ }^{1}$, Nirmal Kumar ${ }^{2}$, Priya Singh ${ }^{3}$ and Pooja Gujjar \\ Received: 25 April 2019 / Accepted: 04 August 2019 / Published online: 28 October 2019 \\ (C) Indian Dairy Association (India) 2019
}

\begin{abstract}
The objective of the present study was to isolate a potential reuterin producing $L$. reuteri strain and check its biopreservation efficacy along with nisin in milk system. A total of 35 lactobacilli isolates were obtained from 12 different sources which included faecal samples from infants, adult humans as well as rats and also human milk. Finally, 13 isolates could be confirmed as Lactobacillus reuteri on the basis of PCR. The 13 $L$. reuteri isolates were then screened for their antimicrobial activity in MRS media with or without glycerol supplementation. It was observed that the supernatant obtained from secondary fermentation from eight $L$. reuteri isolates in glycerolsupplemented media significantly inhibited the growth of majority of bacterial indicators, which was $\mathrm{pH}$ independent. Furthermore the supernatant from the same 8 isolates also exhibited significant antifungal activity against the tested fungal indicators. Based on the RAPD strain profile and reuterin based antimicrobial activity, L. reuteri strain 47 was selected as the most potent isolate for further study. The active units of reuterin in the supernatant of $L$. reuteri 47 were calculated to be $1600 \mathrm{AU} / \mathrm{ml}$. In the milk system, the reuterin system significantly inhibited $E$. coli, but not $P$. acidilactici, nisin on the other hand had significant activity against $P$. acidilactici but not $E$. coli. The combination
\end{abstract}

\footnotetext{
${ }^{1}$ Dairy Microbiology Division, ICAR-National Dairy Research Institute,

Karnal- 132001 (Haryana), India

${ }^{2}$ Biotechnology Department, Graphic Era (Deemed to be University) Dehradun-248 002, Uttarakhand, India

${ }^{3}$ Microbiology Department, Gurukula Kangri Vishwavidyalaya Haridwar-249 407, Uttarakhand, India

Diwas Pradhan $(\square)$

Dairy Microbiology Division

ICAR - National Dairy Research Institute, Karnal- 132001 (Haryana), INDIA

Email : zawidprd@gmail.com; Tel: 91-184-2259169
}

of reuterin and nisin had synergistic effect against $L$. monocytogenes. Hence, the combination of reuterin and nisin can be utilized to target specific pathogens as well as to improve the overall safety of milk and milk products.

Keywords: Antibacterial, Antifungal activity, Lactobacillus reuteri, Milk system, Reuterin

\section{Introduction}

In the recent years, there is an increased demand of minimally processed safe foods with reduced levels of chemical preservatives (Da Costa et al. 2019). This has led to renewed interest in the natural antimicrobials particularly those that are derived from non-pathogenic, food grade Lactic Acid Bacteria (LAB). Many LAB species have been attributed GRAS status (Generally Recognized As Safe) by US-FDA and together with QPS (Qualified presumption of safety) status from EFSA, LAB possess a healthy and natural image (Pradhan et al. 2018). Basically, LAB is a group of fermentative bacteria that are naturally present in many of the food commodities (Ghanbari and Jami, 2013). Many of the LAB are also widely used as starter cultures in the food industry for the production of fermented foods, including dairy (yogurt, cheese), meat (sausages), fish, cereals (bread and beverages such as beer), fruit (malolactic fermentation processes in wine production), and vegetables (sauerkraut, kimchi, silage) (Calo-Mata et al. 2008). During their growth in the food products, $\mathrm{LAB}$ produces numerous antimicrobial metabolites such as organic acids, bacteriocins, diacetyl, fatty acids etc. that exert strong antagonistic activity against many closely related and unrelated microorganisms, including food spoilage organisms and pathogenic bacteria, thereby extending the shelf life of fermented food products.

The biopreservative potential of LAB is well known and many of the earlier reports (Rattanachaikunsopon and Phumkhachorn, 2010) have exclusively elaborated the various antimicrobial substances derived from LAB. Although, the major antagonistic effect of $\mathrm{LAB}$ is due to lowering of $\mathrm{pH}$ by the produced organic acids (lactic acid, acetic acid, formic acid etc.), a number of other metabolites also contribute to the overall biopreservative effect of the LAB (Egan et al. 2016). Among many of the antimicrobials 
produced by the $\mathrm{LAB}$, the bacteriocins have received the most attention chiefly due to its stability, wide spectrum activity and minimum changes to food characteristics (Barbosa et al. 2017). Many bacteriocins from LAB have been isolated and characterized and also classified into different groups (Woraprayote et al. 2016). However only nisin and pediocin PA$1 / \mathrm{AcH}$ are approved to be used in foods, although other bacteriocins mix preparation such as ALTA $^{\mathrm{TM}}$ and MICROGARD ${ }^{\mathrm{TM}}$ are also now commercially available(Goyal et al. 2018). The most limiting factor of bacteriocin activity is its inability to inhibit Gram negative bacteria which limits its antimicrobial action, thereby its application in food systems (Field et al. 2018). On the other hand, some other antimicrobials compounds have been reported from specific LAB organisms that show a wider spectrum of activity against various Gram positive and Gram negative bacteria as well as fungal microorganisms. One such of these antimicrobials having a broad antimicrobial spectrum have been reported from $L$. reuteri strains from diverse niches.

L. reuteri is one of the important species of lactobacilli found as an autochthonous member in the gastrointestinal (GI) tracts of human and many other animals (Casas and Dobrogosz 2000). L. reuteri has also been shown to be a natural component of human milk (Mu et al. 2018). L. reuteri strains are often found in fermented foods (Vollenweider and Lacroix, 2004) as well as marketed as probiotic supplements. The strains of $L$. reuteri possess a unique property of anaerobically converting glycerol into a potent, broadspectrum antimicrobial substance, termed 3hydroxypropionaldehyde (3-HPA), which is also referred to as reuterin (Axelsson et al. 1989). Reuterin (3hydroxypropionaldehyde) is an aldehyde with antimicrobial activity towards a broad spectrum of food-borne pathogens and spoilage organisms, including Gram-positive and Gram-negative bacteria, yeast, molds, and protozoa (Siedler et al. 2019). The compound has a high potential as a food biopreservative since it is water soluble, resistant to heat, active at a wide range of $\mathrm{pH}$ values and resistant to proteolytic and lipolytic enzymes (ElZiney et al. 2000). The antimicrobial efficacy of reuterin has been demonstrated against many of the common food borne spoilage and pathogenic organisms such as Listeria monocytogenes, E. feacalis, Staphylococcus aureus, Peudomanasaeruginosa, Salmonella typhi and Escherichia coli in various food matrices such as milk, dairy products and cottage cheese (Arques et al. 2011). In all these studies, the reuterin system has been shown to have a higher antimicrobial activity than bacteriocins compounds and other non-bacteriocins antimicrobial compounds (Arqués et al. 2008). Therefore, the incorporation of reuterin in food products offers a superior alternative for improving the safety and quality of foods along with reduction in the addition of chemical preservatives (Vollenweider et al. 2003). Hence, the present work was carried to isolate potential reuterin producing L. reuteri strains from different niches and investigate its inhibitory role in milk system.

\section{Materials and Methods}

\section{Bacterial strains and culture conditions}

L. reuteri strain ATCC55730 from Biogaia (Sweden) was used as standard $L$. reuteri strain for the identification of $L$. reuteri isolates. The bacterial as well as fungal target strains used were E. coli K12, Listeria monocytogenes ATCC13932, E. feacalis NCDC135, Serratia marcescens ATCC 13880, S. typhi NCDC 113, Yersinia enterocolitica ATCC130715, Klebsiella pneumonia NCDC 138 and Pediococci acidilactici LB42, and Rhodotorula glutinis NCDC 389, Pencillium roqueforti ATCC 10110 and Aspergillus niger NCDC 267, respectively, for the antimicrobial activity testing of $L$. reuteri isolates. All the bacterial cultures (excluding $P$. acidilactici LB42) were grown in Brain Heart Infusion Broth (BHI, Himedia, India) and P. acidilactici LB42 and $L$. reuteri strains were grown in deMann Rogosa Sharpe broth (MRS; Himedia, India) at $37^{\circ} \mathrm{C}$ for $24-48$ hours. The fungal cultures were grown in Yeast extract Peptone Dextrose broth (YPD; Himedia, India) at $25^{\circ} \mathrm{C}$ for 3-5 days. The fungal cultures were maintained in YPD agar slant at $4{ }^{\circ} \mathrm{C}$, whereas the bacterial strains were maintained as $15 \%$ glycerol stock cultures at $-20^{\circ} \mathrm{C}$ in BHI and MRS broth. The organisms were propagated twice before their use in the experiments.

\section{Sample collection and bacterial isolation}

The samples for L. reuteri isolation were collected locally from Karnal area, Haryana (India). Infant faecal samples, human milk, adult human faeces and rat faecal samples were collected with the help of sterile swabs in sample box containing MRS broth and immediately incubated at $37^{\circ} \mathrm{C} / 2-3 \mathrm{~h}$ for primary enrichment. The samples were then serially diluted in sterile normal saline and appropriate dilutions were plated out in modified MRS medium. Since lactobacilli are known to be intrinsically resistant to antibiotic vancomycin, hence, vancomycin was added in media component to make it selective for lactobacilli growth. The plates were incubated at $37^{\circ} \mathrm{C}$ for $24-48 \mathrm{~h}$. Typical cream coloured, rice shaped colonies were picked up randomly and transferred to MRS broth. After growth at $37^{\circ} \mathrm{C}$ for $24-36 \mathrm{~h}$, the cultures were tested for purity and morphology by microscopic examination.

\section{Identification of bacterial isolate}

The isolated cultures were examined for their morphology by Gram staining and catalase reaction as per standard methods. The tentatively identified lactobacilli isolates based on Gram's staining and catalase test characteristics were further subjected to molecular characterization for confirmation of $L$. reuteri species. All the isolates were grown for 10 to $12 \mathrm{~h}$ in MRS broth at $37^{\circ} \mathrm{C}$ and the genomic DNA was extracted by the method of Pospiech and Neumann (1995). PCR was carried out using primer pair Lreu 1 (CAGACAATCTTTGATTGTTTAG) and Lreu4 (GCTTGTTGGTTTGGGCTCTTC) specific against $L$. reuteri using 
conditions as described by Song et al. (2000). The primers were custom synthesized from Sigma, New Delhi, India.

\section{Preparation of Cell Free Culture Supernatants (CFCSs)}

The preparation of CFCSs from isolated L. reuteri strains were carried out according to the method described by Luthi-Peng, 2002. Briefly, a single colony with typical morphology from the MRS agar plates was inoculated into MRS broth and incubated anaerobically (Gas pack system, Himedia, India) at $37^{\circ} \mathrm{C}$ for $20 \mathrm{~h}$. After incubation, the culture was centrifuged (refrigerated centrifuge, Precision Biotek Instruments Pvt. Ltd., Delhi) at 4,000 $\mathrm{x} g$ for $10 \mathrm{~min}$ at $4^{\circ} \mathrm{C}$. The supernatant was filter-sterilised through a 0.2 ìm syringe filter (Millipore, India) to remove any remaining cells. The cell pellet was used for secondary fermentations to prepare supernatants with MRS supplemented with glycerol (360 $\mathrm{mM})$. The supernatant obtained from secondary fermentation of glycerol-MRS was designated asMRS-Gly, while the supernatant derived from MRS broth was designated as MRSB. These supernatants were kept for a maximum of one month. Finally, the $\mathrm{pH}$ values of the above supernatants were determined using a portable $\mathrm{pH}$ meter ( $\mathrm{pH}$ pen KS732, Shindengen; Japan). The $\mathrm{pH}$ adjustments of the supernatants were carried out by neutralizing them to $\mathrm{pH} 6.5$ using $0.1 \mathrm{M}$ sodium hydroxide at room temperature prior to testing their antimicrobial activity.

\section{Antimicrobial activity of $L$. reuteri isolates}

The agar-well diffusion method was used to check the antibacterial activity of CFCSs derived from isolated cultures of L. reuteri as mentioned in Goyal et al. (2018). Briefly, $10^{6} \mathrm{cfu}$ of target bacteria in its active growth were inoculated to $8 \mathrm{ml}$ of melted and cooled $\left(45^{\circ} \mathrm{C}\right)$ soft agar tubes $(1 \% \mathrm{w} / \mathrm{v})$ of BHI and poured on to the solidified and dried (one day at $37^{\circ} \mathrm{C}$ ) TGE agar plates. The inoculated soft agar was allowed to properly solidify before punching out wells of $6 \mathrm{~mm}$ diameter from agar plates with the help of cork borer. The agar buttons were removed and the base of the wells was sealed by molten soft TGE agar. The drilled wells in the agar plates were then filled with CFCSs $(100 \mu \mathrm{l})$ and the plates were kept at $4^{\circ} \mathrm{C}$ for $3-4 \mathrm{~h}$ to facilitate the diffusion of CFCSs. The plates were then incubated at $37^{\circ} \mathrm{Cand}$ the antimicrobial activity was recorded as the zone of inhibition extending laterally from the edges of wells.

The antifungal activity of CFCSs was performed using agar overlay assay (Voulgari et al. 2010). Briefly, $5 \mu$ l of active cultures of $L$. reuteri isolates were spotted on MRS (with glycerol) agar plates and incubated for $48 \mathrm{~h}$ at $37^{\circ} \mathrm{C}$ under anaerobic conditions. Subsequently, an aliquot of $100 \mu$ lof fungal spores (corresponding to $10^{4}$ spores) were transferred to melted and cooled $\left(45^{\circ} \mathrm{C}\right) \mathrm{YPD}$ soft agar. The $\mathrm{pH}$ of soft agar was adjusted to 4.0 with sterile tartaric acid before pipetting the soft agar on to the culture agar plates. The soft agar was pipetted on the surface of petridish drop wise surrounding the culture spots. The soft agar was allowed to solidify and the plates were incubated at $30^{\circ} \mathrm{C}$ for $3-5$ days depending on the fungal growth rate. The antifungal activity of the culture was measured in terms of the diameter of zoneof inhibition at the end of incubation period.

\section{RAPD-PCR of $L$. reuteri isolates}

Random amplified polymorphic DNA (RAPD)-PCR was performed on the $L$. reuteri isolates with antimicrobial activity to determine the genetic relatedness and exclude replicates among the isolates. A total of 5 random RAPD primers (Weiss5, Weiss1, 275, OPPGandOPAP-1) were first tested for RAPD band pattern, out of which OPAP-1 (AACTGGCCCC)(Singh et al. 2017) was selected based on reproducibility and number of bands. RAPD-PCR was carried out following standard PCR protocol and the PCR amplified products obtained were electrophoresed on $1.5 \%$ agarose gels at low voltage of $60 \mathrm{~V}$ for $3 \mathrm{~h}$ (Singh et al. 2017).

\section{Activity units of Cell Free Culture Supernatant (CFCS)}

The active units of CFCS of the most potent culture was quantified by MIC method using microtiter plate method against E. coli $\mathrm{K} 12$ as described by Chung et al. (1989). Overnight grown culture of E. coli $\mathrm{K} 12$ was harvested, washed twice with phosphate buffer ( $\mathrm{pH} 7.2,50 \mathrm{mM}$ ), suspended in the same buffer, and diluted to an $\mathrm{A}_{420}$ of 0.2 OD measured using spectrophotometer. This suspension was diluted $1 / 10$, which corresponds to about $1 \times 10^{6} \mathrm{cfu} / \mathrm{ml}$. The diluted suspension $(0.1$ $\mathrm{ml}$ ) was used to inoculate $0.2 \mathrm{ml}$ of serial dilutions of CFCSs diluted in Muller-Hinton medium (Himedia, India Laboratories). The microplate was incubated at $37^{\circ} \mathrm{C}$ for $12 \mathrm{~h}$ and examined accordingly for growth. The active units in the CFCSs was defined as the reciprocal of the highest dilution that did not permit visible growth of the indicator strain and expressed as Arbitrary Units (AU).

\section{Antimicrobial activity in milk system}

The inhibitory action of reuterin alone (CFCS) or in combination with a bacteriocin (nisin) was finally tested in milk system against some model food microorganisms viz. E. coli K12, Pediococcus acidilactici LB42 and Listeria monocytogenes ATCC 13932. These indicator organisms were selected since $E$. coli is sensitive to reuterin, while LB42 strain is nisin sensitive while Listeria is sensitive to both the tested antimicrobial substances, hence this would allow testing the synergistic effect between the two antimicrobials and also whether the drawback of one can be fulfilled by another.

Stock solutions of nisin (10000IU/ml) (Nisaplin, Danisco, India) were prepared in $0.02 \mathrm{~N} \mathrm{HCl}$ and stored at $-20^{\circ} \mathrm{C}$. The stock solution of nisin was diluted accordingly and $100 \mathrm{IU} / \mathrm{ml}$ was added into the milk system. The CFCSs was then added at an estimated final activity of $16 \mathrm{AU} / \mathrm{ml}$. Cells (of the target bacteria) of the early stationary phase were harvested and $100 \mu$ l were separately 
inoculated into flasks containing $100 \mathrm{ml}$ of reconstituted skim milk (11\% total solids). The seeded milk with each bacterial strain with no addition of reuterin or nisin served as control. All the flasks were then subsequently incubated at $37^{\circ} \mathrm{C}$ for $24 \mathrm{~h}$. The microbiological counts of the entire flask were determined at $0 \mathrm{~h}$ and subsequently after 4 and $24 \mathrm{~h}$ on BHI and MRS medium agar plates.

\section{Statistical analysis}

The data obtained in this study was presented as mean \pm standard deviation and analyzed statistically with the GraphPad Prism 5.0 software. The statistical significance among different antimicrobials and its combination in milk system was compared by one way ANOVA following Tukey's post hoc test and considered significant at P d" 0.05 .

\section{Results and Discussion}

\section{Isolation and Identification of $L$. reuteri strains}

Lactic acid bacteria are natural inhabitants of nutrient rich niches, in particular L. reuteri are common residents of the gastrointestinal tract (GIT) of diverse mammals such as humans, pigs, mice, rats and many species of birds (Frese et al. 2011).L. reuteri have been isolated from the gastrointestinal tract of human, pigs etc. (Hou et al. 2015). Therefore, we obtained a number of samples comprising of infant faeces, adult faeces, breast milk and rat faecesin an attempt to obtain some potential strains of Lactobacillus reuteri with broad spectrum antimicrobial activity.
From the plated MRS samples (with vancomycin), a total of 113 random colonies were picked based on colonial morphology (cream colour, oval shape), and used for further analysis. Based on the phenotype screening test (standard Gram staining procedure and catalase test), a total of 35 isolates were found to be Gram positive rods and catalase negative and were presumed to be Lactobacillus cultures. Further, the L. reuteri confirmation was carried out for the 35 tentative Lactobacillus isolates with species specific PCR using primer pair Lreu 1/Lreu 4. Thirteen isolates out of the 35 tested isolates gave a specific amplification product of $303 \mathrm{bp}$ for L. reuterin specific PCR (Fig. 1). Earlier, it has been reported that $L$. reuteri is one of the most abundant species present in the gut of pigs, rodents, and chickens, (Abbas et al. 2000), however the prevalence of $L$. reuteri is much lower in human adults, where the species is only occasionally found (Walter 2008). Similarly in our study no L. reuteri isolate could be obtained from human adult feces, and out of the final 13 confirmed $L$. reuteriisolates, 10 were from breast milk, 2 from infant faeces and 1 from rat faeces. The 13 PCR confirmed isolates of $L$. reuteri were then subjected to further analysis.

\section{Antimicrobial activity of Cell Free Culture Supernatants (CFCS) against selected indicator organisms}

Some strains of $L$. reuteri can produce reuterin from anaerobic conversion of glycerol (Siedler 2019). Reuterin is a potent antimicrobial compound that has a wide spectrum of antimicrobial activity surpassing various kinds of bacteria, fungi, protozoa etc. (Axelsson et al. 1989). However, the reuterin producing capacity is highly variable among different strains of $L$. reuteri

Table 1 Antimicrobial activity of $L$. reuteri isolates against Gram negative and positive bacterial indicators by agar well diffusion assay

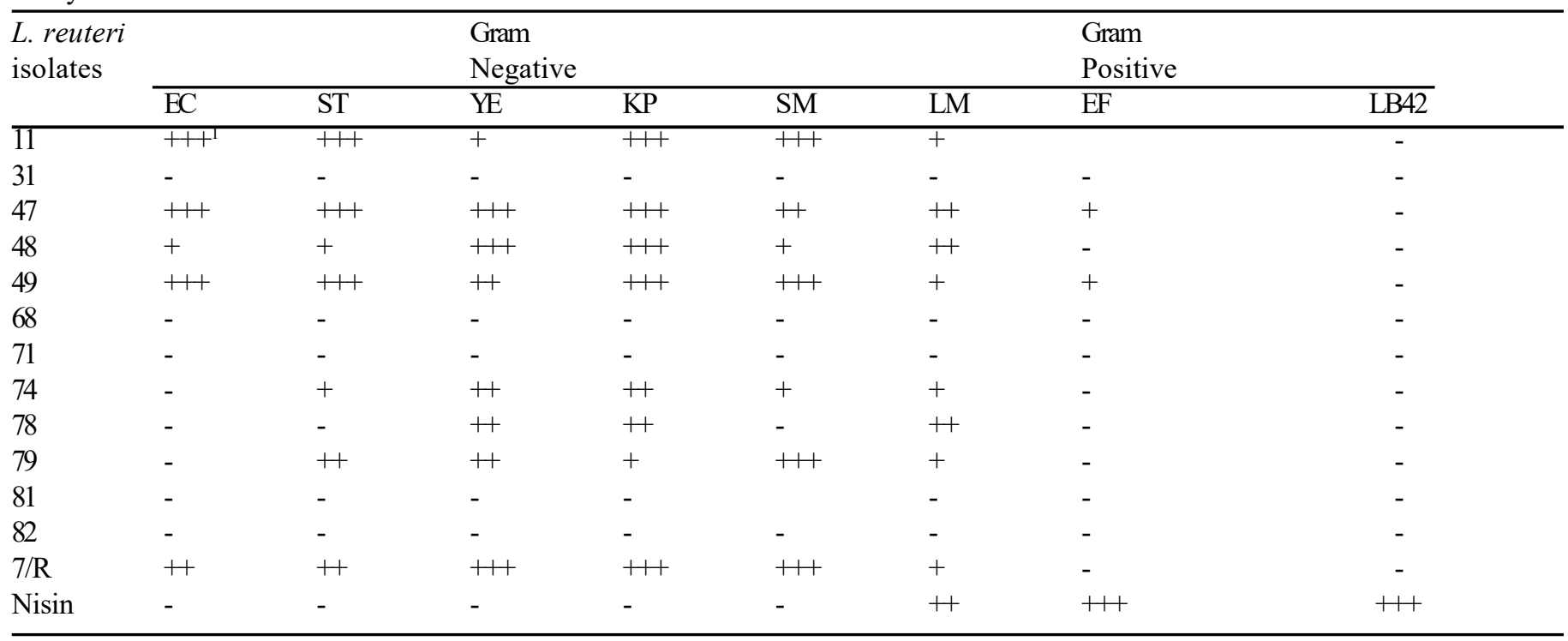

'Zone of inhibition: $+++:>15 \mathrm{~mm},++: 10-15 \mathrm{~mm},+:<10 \mathrm{~mm}$.

$\mathrm{EC}=$ Escherichia coli, $\mathrm{ST}=$ Salmonella typhi, $\mathrm{YE}=$ Yersinia enterocolitica, $\mathrm{KP}=$ Klebsiella pneumonia $; \mathrm{SM}=$ Serratia marcescens $;$ $\mathrm{LM}=$ Listeria monocytogenes; $\mathrm{EF}=$ Enterococcus feacalis $; \mathrm{LB} 42=$ Pediococciacidilactici 
Fig. 1 Species specific PCR of isolated Lactobacillus reuteri strains using Lreu-1 and Lreu-4 primer pair; Lane M: 100 bp DNA Marker; L1: $L$. reuteri ATCC 55730; L2: 47; L3: 49; L4: 68; L5: 74; L6: 78 L7: 7/R.

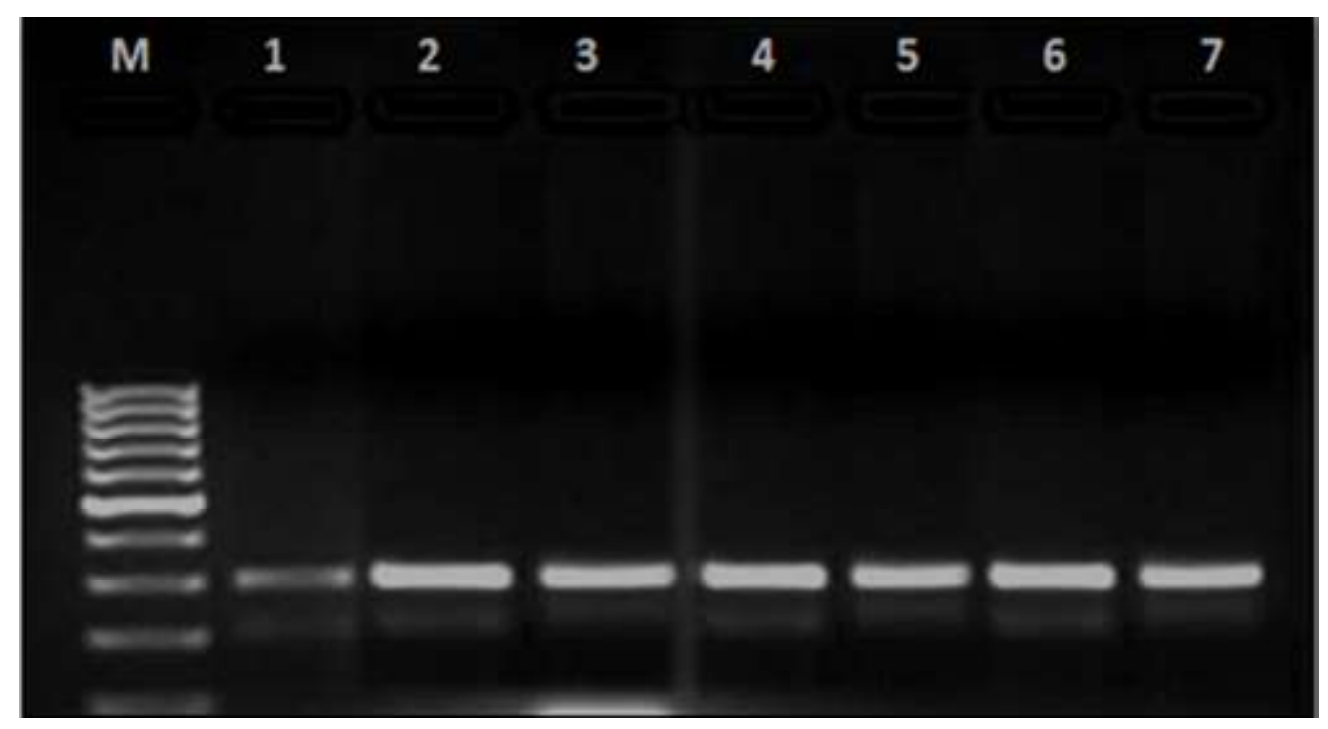

Table 2 Antifungal activity of $L$. reuteri isolates against fungal indicators by agar overlay assay

\begin{tabular}{|c|c|c|c|c|c|c|c|c|c|c|c|c|c|}
\hline \multirow{2}{*}{$\begin{array}{l}\text { Fungal } \\
\text { indicators }\end{array}$} & \multicolumn{13}{|c|}{ L. reuteri } \\
\hline & 11 & 31 & 47 & 48 & 49 & 68 & 71 & 74 & 78 & 79 & 81 & 82 & $7 / \mathrm{R}$ \\
\hline A. niger & $+1+$ & + & $+1+$ & - & + & - & + & - & + & $+1+$ & + & - & + \\
\hline R. glutinis & $+1+$ & + & $+1+$ & + & + & + & + & + & + & $+1+$ & + & + & $+1+$ \\
\hline
\end{tabular}

+ Less than $20 \mathrm{~mm}$; ++ 20-35 mm; +++Greater than $35 \mathrm{~mm}$

due to variations in gene expression of associated enzymes (Spinler et al. 2008). We screened the 13 identified L. reuteri strains for its antimicrobial activity against 8 different bacterial indicators that included important food pathogens. Both the neutralized as well as non-neutralized forms of CFCS derived from MRS broth fermented with glycerol supplementation (MRSGly) as well as without glycerol supplementation (MRSB) were used to test the antimicrobial activity. The antimicrobial activity was observed in both MRS-Gly as well as MRSB, however the inhibitory effect of MRSB was not evident in its $\mathrm{pH}$ adjusted counterparts. $L$. reuteri being a $\mathrm{LAB}$ secrete organic acids such as lactic acid, acetic acid etc. as a primary fermentation product. These are strong antimicrobial agents particularly in a relative low $\mathrm{pH}$ environment, which may have contributed the antimicrobial activity observed in case of MRSB, since the activity could not be replicated in its $\mathrm{pH}$ neutralized counterpart. On the other hand, CFCS (neutralized) from MRS-Gly derived from 8 strains out of the 13 identified L. reuteri strains exhibited good antibacterial activity against majority of the indicator organisms (Table 1 and Fig. 3). The activity observed in this case can be attributed to reuterin action since reuterin is active at wide range of $\mathrm{pH}$ values including neutral $\mathrm{pH}$ (Garde et al. 2016). The highest antimicrobial activity was observed in the neutralized CFCS derived from $L$. reuteristrain $11,47,49$ and $7 \mathrm{R}$. The CFCS of these L. reuteri strains were able to inhibit both Gram negative as well as Gram positive bacterial indicators. Moderate activity was seen in $L$. reuteri strain $48,74,78$ and 79 , while very negligible or no activity was observed in L. reuteri strain $31,68,71,82$ and 81 strains. However, none of the L. reuteri isolates had any significant inhibitory effect against Enterococcus faecalis and Pediococcus acidilactici. This is not surprising as it has been reported that reuterin affects lactic acid bacteria to a lesser degree and relatively higher concentration of reuterin is required to inhibit the growth of LAB (Axelsson et al. 1989).

\section{Screening of $L$. reuteri isolates for antifungal activity}

The most important attribute of reuterin system is its wide spectrum antimicrobial activity, which gives it a very important prospect of being an excellent biopreservative molecule. In addition to inhibiting a wide range of bacterial indicators, the reuterin system has also been shown to demonstrate wide antifungal activity (Schnurer and Magnusson, 2005). Here, the antifungal activity of the $L$. reutericultures as well as its supernatant was checked by agar overlay and spot on lawn assay on mMRS and MRS media, respectively. Out of the two methods, the agar overlay assay using $L$. reuteri culture streak in mMRS media was found to be more suitable for assessing the antifungal activity of $L$. reuteri strains, hence the antifungal activity testing of the test strains was carried out with the agar overlay assay. From the agar overlay assay, it was observed that the indicator organism Rhodotorula glutinis was heavily inhibited by all the $L$. reuteri isolates and very low growth of the fungal spores was observed in the agar plates containing the L. reuteri cultures, 


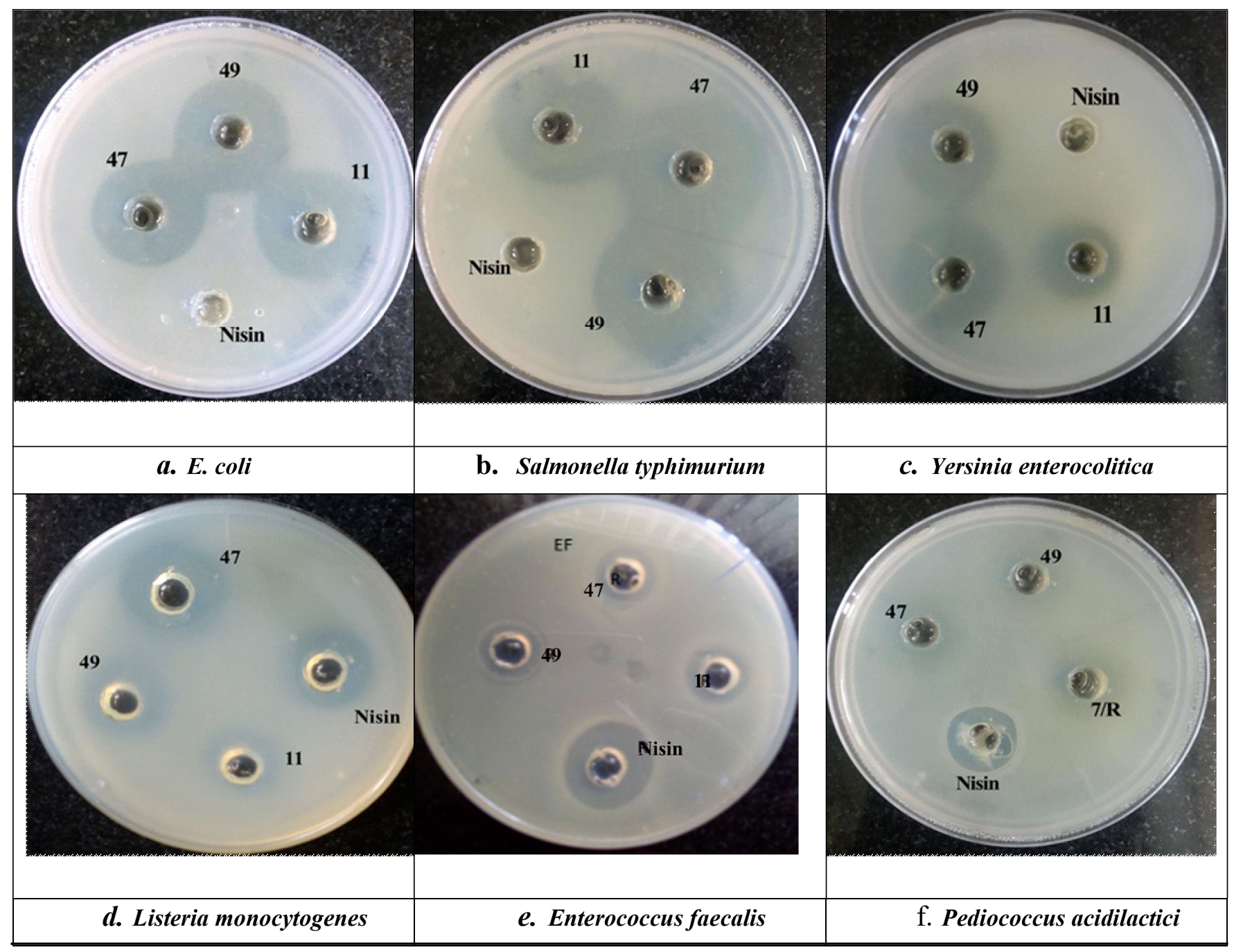

Fig. 2 Antimicrobial activity of CFCSs of $L$. reuteri strains and nisin against different indicator strains (Gram positive and Gram negative) by agar well assay

although, we could observe proper growth of $R$. glutinis in the agar plate without any L. reuteri culture spots (Table2). Similarly, Penicillium roqueforti also showed little growth in the presence of most of the isolated $L$. reuteri cultures. The mold $A$. niger was the most resistant fungus to the inhibitory activity of $L$. reuteriisolates and only strain 47,79 and 11 showed significant inhibition, while other strains showed little or no inhibition against A. niger. Earlier, it has been demonstrated by Vimont et al. 2019, that reuterin at concentration $6.9 \mathrm{mM}$ can display strong fungicidal activity against a host of food fungi including the fungal indicators tested in this study. The concentration reported is generally formed in the common method of reuterin production by $L$. reuteri strains. Hence, we can presume that the isolated $L$. reuteri strains in the study were able to produce enough reuterin in the medium in order to inhibit the growth of tested fungal indicators.

\section{RAPD analysis of $L$. reuteri strains}

A total number of five arbitrary primers were tested with RAPDPCR with DNA extracts from pure cultures of $8 \mathrm{~L}$. reuteri isolates showing antimicrobial activity. The RAPD primer, OPAP-1 for $L$. reuteri was able to produce more than four scorable bands and was selected for further analysis. The profile generated from RAPD-PCR of the 8 L. reuteri cultures generated between 8 to 10 DNA bands ranging from 100 to $>1500 \mathrm{bp}$ (Fig. 3). The strains isolated from infant faeces (Lane 2) showed similar pattern as did the strains isolated from human milk (Lanes 4, 5, 7, and 8), although variation in the antimicrobial activity was observed between the strains. Previously, Singh et al. (2017) also reported that the strain types resulting from RAPD-PCR do not necessarily segregate based on metabolic activity. On the contrary, the strain (Lane 1) isolated from rat gave distinct RAPD profile from other strains from human milk. Although, Ganzle and Yogel, (2003) have previously described that not all $L$. reuteri strains from different sources could be discriminated on account of their RAPD-PCR patterns with one arbitrary primer, we were able to detect the 
Fig. 3 RAPD profiles of $L$. reuteri strains showing antimicrobial activity generated against RAPD primer OPAP-1. Lanes M: 100 bp DNA ladder; L1: $7 /$ R;L2: 11;L3: 74; L4:49; L5: 79; L6: 47; L7: 78; L8: Lb48

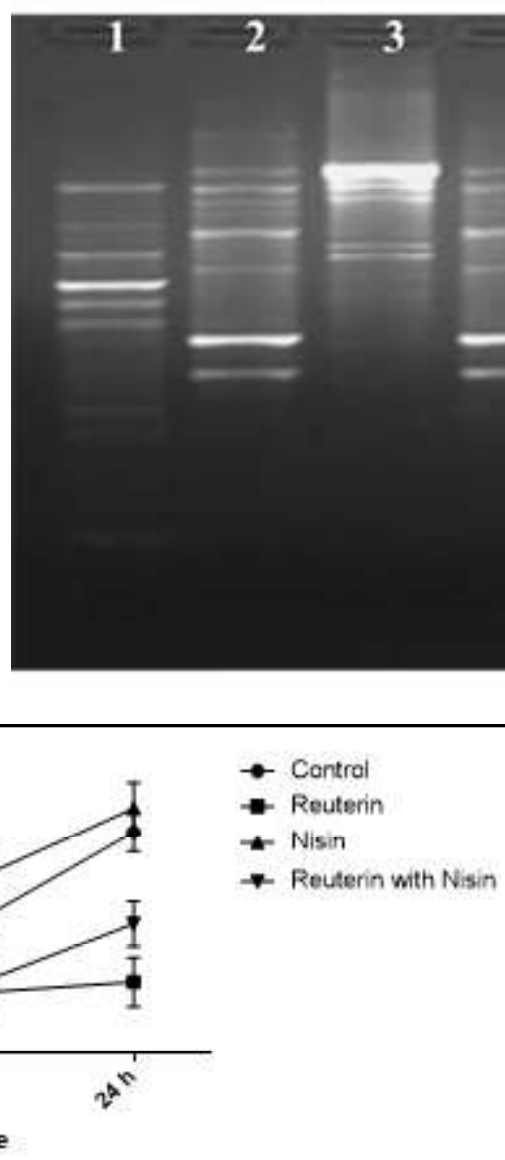

a. E. coli $\mathrm{K} 12$

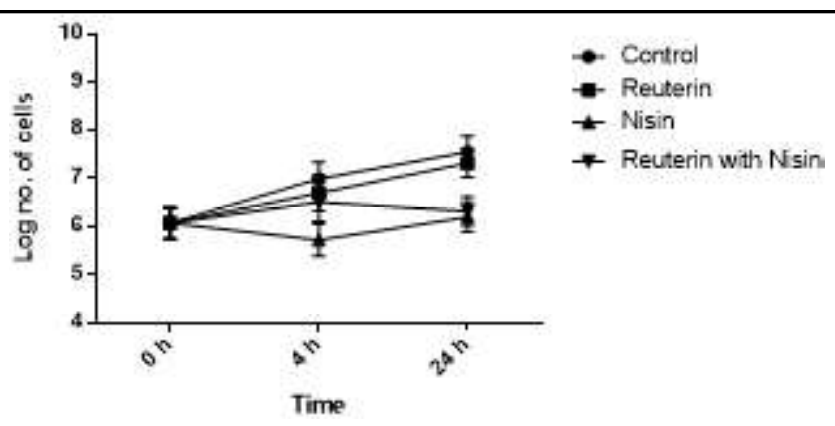

b. Pediococcus acidilactici LB 42

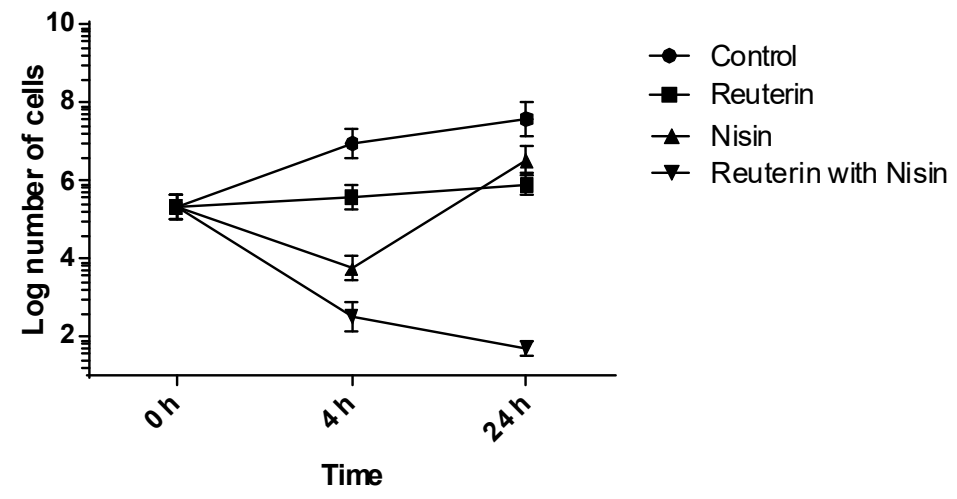

\section{c. Listeria monocytogenes}

Fig. 4 Log counts (cfu/ml) of Gram-positive and Gram-negative pathogens in milk without antimicrobial agents(C), with reuterin, with nisin and their combination $(\mathrm{R}+\mathrm{N})$ after incubation at $37^{\circ} \mathrm{C}$ for $4 \mathrm{~h}$ and $24 \mathrm{~h}$.

strain replicates and overall 4 group or strain type was obtained from the tested 8 L. reuteri strains.

\section{Activity units of the most potent $L$. reuteri strain 47}

Based on the antimicrobial (antibacterial and antifungal) activity and RAPD profile, $L$. reuteri 47 was selected as the most potent isolate and used for further study. The active units of reuterin present in the cell free culture supernatant (CFCS) of the L. reuteri 47 was calculated by MIC determination against $E$. coli $\mathrm{K} 12$ by microtitre plate assay. Serial dilutions of CFCS of L. reuteri 47 were prepared in microtitre plate and activity unit of CFCS was obtained against defined number of cells of $E$. coli in the microtiter plate. After overnight incubation at $37^{\circ} \mathrm{C}$, growth was observed in the fifth well pertaining to $16 \mathrm{x}$ dilution of the stock CFCSs. No visible growth was observed in the fourth dilution (pertaining to 
$8 \mathrm{x}$ dilution), accordingly the activity unit of the CFCS was tabulated as $1600 \mathrm{AU} / \mathrm{ml}$.

\section{Antibacterial activity of reuterin from $L$. reuteri47alone or in combination with nisin in milk system}

As mentioned earlier, biopreservation of processed foods is an attractive area particularly using metabolites of LAB due to its safe nature. However, much work has been focussed on the proteinaceous bacteriocins which due to its cationic nature are unable to penetrate the LPS layer of Gram negative bacteria and reach the target site i.e. cell wall (Field et al. 2018). However, Gram negative bacteria such as coliforms and other members of enterobacteriaceae family consist of the major spoilage and pathogenic microflora in food products including milk and milk products. Contrary to the bacteriocins, the reuterin system acts by modifying the thiol groups in proteins and small molecules of the target microorganisms, therefore, are able to inhibit a wide spectrum of both Gram positive and negative bacteria including fungal targets (Siedler et al. 2019). However, reuterin has been demonstrated to have weak effect on LAB, probably due to its antioxidant system, hence, a combination of reuterin and nisin was tested in this study for best antimicrobial action. Furthermore, the antimicrobial action of these molecules have been observed to display variable antimicrobial action in different food systems, possibly due to interactions with food components, hence it is very important to test the antimicrobial action of any antimicrobial system in the real food system.

In this study, the inhibitory action of reuterin $(16 \mathrm{AU} / \mathrm{ml})$, nisin $(100 \mathrm{IU} / \mathrm{ml})$ and their combination on certain bacterial indicators viz. E. coli $\mathrm{K} 12$, P. acidilactici $\mathrm{LB} 42$ and Listeria monocytogenes were tested in sterile milk system as shown in Fig. 4.In case of $E$. coli $\mathrm{K} 12$, as anticipated the presence of reuterin in milk had a significant bacteriostatic effect on the growth of $E$. coli, which did not demonstrate any net increase in counts even after $24 \mathrm{~h}$ of incubation at $37^{\hat{i}} \mathrm{C}$ (Fig. 4a). On the other hand, nisin had negligible effect and the growth of $E$. coli in its presence was similar to the control milk sample with no additive. Furthermore, no synergistic effect was seen when both the antimicrobials were mixed, rather nisin had a slight hindering effect on the reuterin action and the numbers increased after an initial reduction. Conversely, Pediococcus acidilactici LB42 is a bacteriocin sensitive strain (Goyal et al. 2018) and as expected the inhibition was also demonstrated in the milk system wherein LB42 counts decreased initially on nisin exposure and no net growth was observed after $24 \mathrm{~h}$ incubation (Fig. 4b). However no effect of reuterin was observed on LB42. The synergistic effect could only be seen in case of L. monocytogenes. It is well known that both nisin and reuterin have antagonistic activity against $L$. monocytogenes (Arques et al. 2011) which was also seen here in the milk system. In case of reuterin, the activity observed was more bacteriostatic since there was no significant inhibition after $4 \mathrm{~h}$ and even after $24 \mathrm{~h}$ the counts did not variably increase. On the other hand, nisin has bacteriocidal effect on L. monocytogenes since the count had significantly decreased (Fig. 4c) after initial $4 \mathrm{~h}$ incubation, thereafter the growth resumed. The increase of $L$. monocytogenes cells after $24 \mathrm{~h}$ incubation maybe attributed to the regrowth of resistant cells.

Previously, numerous attempts have been made to prepare combinations of nisin with other antimicrobial compounds such as ascorbic acid and EDTA (Sangcharoen et al. 2017; Khan et al. 2015), plant essential oils and ascorbic acid (Campion et al. 2017), EDTA and lysozyme (Bhatia and Bharti, 2015) etc. in order to overcome the drawback of LAB bacteriocins. Although, these formulations were successful in inhibiting Gram negative organisms also, the application of most of these antimicrobials is limited either due to its chemical nature, production cost or interference with the sensory properties of the final product. In this regard, reuterin offers a suitable alternative due to the GRAS status of the producing species, besides being active against a wide target range and physical conditions of temperature, $\mathrm{pH}$ etc. along with the absence of any sensory characteristics. In the present study, reuterin and nisin were able to display synergistic effects against L. monocytogenes, besides overcoming the drawbacks of one another against other target microorganisms in milk system. Apart from displaying efficacy in milk system reuterin have also been positively tested against Listeria monocytogenes in cottage cheese and UHT skim milk (El-Ziney and Debevere, 1998), besides controlling Clostridium spp. spores growth in dairy products (Avila et al. 2014). The target site of reuterin system is different from that of nisin. Due to the differences in target sites of reuterin and nisin, the combination can cause multiple cell injuries in the target cell that could inhibit even the resistant strains and also prevent the emergence of resistance (Arques et al. 2011). However, very limited studies have attempted to study the efficacy of these antimicrobial substances against the mixed microflora that exist in natural food products rather than spiked food samples. Hence, even in case of the reuterin and nisin combination tested in the present study, it is important to check the activity in the natural mixed microbial population that exists in different foods before building applications for specific food systems.

\section{Conclusions}

In the present study, reuterin and nisin in the tested concentration could effectively inhibit their individual targets, in addition to showing a synergistic activity against a common target such as Listeria monocytogenes in milk system. Hence, it may be concluded here that reuterin can form effective combination with bacteriocins such as nisin in order to target different microbial food contaminants. The suitability of the combination should also be tested in different food matrices in order to utilise as an effective biopreservative. However, further studies needs to be carried out on its toxicity and also efficacy against the natural 
microflora persisting in different food systems in order to use the combination as an effective food biopreservative.

\section{Acknowledgments}

The author duly acknowledges the Director, ICAR-National Dairy Research Institute, Karnal, Haryana (India) for providing all the necessary facilities for carrying out the present work.

\section{References}

Abbas Hilmi HT, Surakka A, Apajalahti J, Saris PE (2007) Identification of the most abundant Lactobacillus species in the crop of 1- and 5week-old broiler chickens. Appl Environ Microbiol 73: 7867-7873

Arques JL, Rodríguez E, Nunez M, Medina M (2008a) Antimicrobial activity of nisin, reuterin, and the lactoperoxidase system on Listeria monocytogenes and Staphylococcus aureus in cuajada, a semisolid dairy product manufactured in Spain. J Dairy Sci. 91: 70-75

Arques JL, Rodrýguez E, Nunez M, Medina M (2011) Combined effect of reuterin and lactic acid bacteria bacteriocins on the inactivation of food-borne pathogens in milk. Food Control 22: 457-46

Avila M, Gomez-Torres N, Hernández M, Garde S (2014) Inhibitory activity of reuterin, nisin, lysozyme and nitrite against vegetative cells and spores of dairy-related Clostridium species. Int J Food Microbiol 172: $70-75$

Axelsson LT, Chung TC, Dobrogosz WJ, Lindgren SE (1989) Production of a broad spectrum antimicrobial substance by Lactobacillus reuteri. Microb Ecol Health Dis 2: 131-136

Barbosa AAT, Mantovani HC, Jain S (2017). Bacteriocins from lactic acid bacteria and their potential in the preservation of fruit products. Crit Rev Biotechnol 37: 852-864

Bhatia S, Bharti A (2015) Evaluating the antimicrobial activity of Nisin, Lysozyme and Ethylenediaminetetraacetate incorporated in starch based active food packaging film. J Food Sci Technol 52: 35043512

Calo-Mata P, Arlindo S, Boehme K, de Miguel T, Pascoal A, BarrosVelazquez J (2008) Current applications and future trends of lactic acid bacteria and their bacteriocins for the biopreservation of aquatic food products. Food and Bioproc Tech 1: 43-63

Campion A, Morrissey R, Field D, Cotter PD, Hill C, Ross RP (2017) Use of enhanced nisin derivatives in combination with food-grade oils or citric acid to control Cronobacter sakazakii and Escherichia coli O157: H7. Food Microbiol 65: 254-263

Casas IA, Dobrogosz WJ (2000) Validation of the Probiotic Concept: Lactobacillus reuteri confers broad-spectrum protection against disease in humans and animals. Microb Ecol Health Dis 12: 247285

Hou C, Zeng X, Yang F, Liu H, Qiao S (2015) Study and use of the probiotic Lactobacillus reuteri in pigs: A review. J Anim Sci Biotechnol 6: 14

Chung TC, Axelsson L, Lindgren SE, Dobrogosz WJ (1989) In vitro studies on reuterin synthesis by Lactobacillus reuteri. Microb Ecol Health Dis 2: $137-144$

Da Costa RJ, Voloski FL, Mondadori RG, Duval EH, Fiorentini AM (2019) Preservation of meat products with bacteriocins produced by lactic acid bacteria isolated from meat. J Food Qual. https://doi.org/10.1155/ 2019/4726510

Egan K, Field D, Rea MC, Ross RP, Hill C, Cotter PD (2016) Bacteriocins: Novel Solutions to Age Old Spore-Related Problems? Front Microbiol 7
El-Ziney MG, Debevere JM (1998) The effect of reuterin on Listeria monocytogenes and Escherichia coli $\mathrm{O} 157$ : $\mathrm{H} 7$ in milk and cottage cheese. J Food Prot 61: 1275-1280

El-Ziney MG, Debevere JM, Jakobsen M (2000) Reuterin. In: Naidu, A.S. (Ed.), Natural Food Antimicrobial Systems. CRC Press, Boca Raton, FL., USA, pp. 567e588

Field D, Ross RP, Hill C (2018) Developing bacteriocins of lactic acid bacteria into next generation biopreservatives. Curr Opin Food Sci 20: $1-6$

Frese SA, Benson AK, Tannock GW, Loach DM, Kim J, Zhang M (2011) The evolution of host specialization in the vertebrate gut symbiont Lactobacillus reuteri. PLoS Genet 7:1001314

Ganzle MG, Vogel RF (2003) Contribution of reutericyclin production to the stable persistence of Lactobacillus reuteri in industrial sourdough fermentation. Int J Food Microbiol 80(1): 31-45

Garde S, Gomez-Torres N, Delgado D, Gaya P, Avila M (2016) Influence of reuterin-producing Lactobacillus reuteri coupled with glycerol on biochemical, physical and sensory properties of semi-hard ewe milk cheese. Food Res Int 90: 177-185

Ghanbari M, Jami M (2013) Lactic acid bacteria and their bacteriocins: a promising approach to seafood biopreservation. In Lactic acid bacteria-R \& D for food, health and livestock purposes. InTech

Goyal C, Malik RK, Pradhan D (2018) Purification and characterization of a broad spectrum bacteriocin produced by a selected Lactococcus lactis strain 63 isolated from Indian dairy products. J Food Sci Technol 55: 3683-3692

Khan A, Vu KD, Riedl B, Lacroix M (2015) Optimization of the antimicrobial activity of nisin, Na-EDTA and $\mathrm{pH}$ against gramnegative and gram-positive bacteria. Food Sci Tech 61: 124-129

Luthi-Peng Q, Dileme FB, Puhan Z (2000) Effect of glucose on glycerol bioconversion by Lactobacillus reuteri. Appl Environ Microbiol 59: 289-296

Mu Q, Tavella V, Luo XM (2018) Role of Lactobacillus reuteri in Human Health and Diseases. Front Microbiol 9: 757

Pospiech A, Neumann B (1995) A versatile quick preparation of genomic DNA from gram-positive bacteria. Trends Genet 11: 217-218

Pradhan D, Singh R, Tyagi A, Rashmi HM, Batish VK, Grover S (2019) Assessing safety of Lactobacillus plantarum MTCC 5690 and Lactobacillus fermentum MTCC 5689 using in vitro approaches and an in vivo murine model. Regul Toxicol Pharmacol 101: 1-11

Rattanachaikunsopon P, Phumkhachorn P (2010) Lactic acid bacteria: their antimicrobial compounds and their uses in food production. Ann Biol Res 1: 218-228

Sangcharoen N, Klaypradit W, Wilaipun P (2017) Antimicrobial activity optimization of nisin, ascorbic acid and ethylenediaminetetraacetic acid disodium salt (EDTA) against Salmonella enteritidis ATCC 13076 using response surface methodology. Agri Nat Res 51(5):355-364

Schnurer J, Magnusson J (2005) Antifungal lactic acid bacteria as biopreservatives. Trends Food Sci Tech 16: 70-78

Siedler S, Balti R, Neves AR (2019) Bioprotective mechanisms of lactic acid bacteria against fungal spoilage of food. Curr Opin Biotech 56: 138-146

Singh A, Pradhan D, Goyal C (2017) Characterization of antibiotic resistance in dairy Lactococci. Indian J Dairy Sci 70: 324-330

Song YL, Kato N, Liu CX, Matsumiya Y, Kato H, Watanabe K (2000) Rapid identification of 11 human intestinal Lactobacillus species by multiplex PCR assays using group and species- specific primers derived from the 16S-23S rRNA intergenic spacer region and its flanking 23S rRNA. FEMS Microbiol Lett 187: 167-173

Spinler JK, Taweechotipatr M, Rognerud CL, Oub CN, Tumwasorn S, Versalovic J (2008) Human-derived probiotic Lactobacillus reuteri demonstrate antimicrobial activities targeting diverse enteric bacterial pathogens. Anaerobe 14: 166-171 
Vimont A, Fernandez B, Ahmed G, Fortin HP, Fliss I (2019) Quantitative antifungal activity of reuterin against food isolates of yeasts and moulds and its potential application in yogurt. Int J Food Microbiol 289: $182-188$

Voulgari K, Hatzikamari M, Delepoglou A, Georgakopoulos P, LitopoulouTzanetaki E, Tzanetakis N (2010) Antifungal activity of non-starter lactic acid bacteria isolates from dairy products. Food Cont 21: 136142

Vollenweider S, Lacroix C (2004) 3-Hydroxypropionaldehyde: applications and perspectives of biotechnological production. Appl Environ Microbiol 64: 16-27
Vollenweider S, Grassi G, Konig I, Puhan Z (2003) Purification and structural characterization of 3-hydroxypropionaldehyde and its derivatives. J Agri Food Chem 51: 3287-3293

Walter J (2008) Ecological role of lactobacilli in the gastrointestinal tract: Implications for fundamental and biomedical research. Appl Environ Microbiol 74: 4985-4996

Woraprayote W, Malila Y, Sorapukdee S, Swetwiwathana A, Benjakul S, Visessanguan W (2016) Bacteriocins from lactic acid bacteria and their applications in meat and meat products. Meat Sci 120: 118132 